\title{
Desempenho e medidas biométricas de caprinos de diferentes grupos raciais $^{1}$
}

\section{Jakilane Jacque Leal de Menezes $^{2}$, Heraldo Cesar Gonçalves ${ }^{3}$, Marcela Silva Ribeiro ${ }^{4}$, Luciana Rodrigues ${ }^{2}$, Gil Ignácio Lara Cañizares ${ }^{4}$, Brenda Batista Lemos Medeiros ${ }^{5}$, Ana Paula Giassetti ${ }^{5}$}

\author{
1 Parte da dissertação de Mestrado em Zootecnia apresentada pela primeira autora à UNESP. Projeto financiado pela FAPESP. \\ 2 Doutoranda do Programa de Pós-Graduação em Zootecnia, FMVZ, UNESP, Botucatu - SP. \\ ${ }^{3}$ Departamento de Produção e Exploração Animal, FMVZ, UNESP, Botucatu- SP. \\ ${ }^{4}$ Mestrando do Programa de Pós-Graduação em Zootecnia, FMVZ, UNESP, Botucatu- SP. \\ 5 Graduando em Zootecnia, FMVZ, UNESP, Botucatu - SP.
}

RESUMO - Objetivou-se avaliar o desempenho de cabritos de três grupos raciais. Foram considerados nas avaliações o peso, o ganho de peso, as medidas biométricas, a conversão alimentar e o custo de produção relativo à alimentação. Utilizaram-se 41 cabritos, machos e fêmeas, de três grupos raciais (A - Alpino; 1/2 BA - 1/2 Boer + 1/2 Alpino; e 3/4 BA - 3/4 Boer + 1/4 Alpino), avaliados aos 60, 90 e 120 dias em sistema de confinamento. As médias foram comparadas por análise de variância utilizando-se o teste Tukey. O desempenho não diferiu entre os grupos raciais. O escore corporal foi maior nos animais $3 / 4 \mathrm{BA}$, o que pode representar vantagem econômica ao produtor se esses animais forem comercializados vivos. Com o avanço da idade, houve aumento do comprimento corporal, da altura anterior, da altura posterior, do perímetro torácico, do perímetro da perna, da largura da garupa e da largura do peito. Os machos foram superiores às fêmeas quanto às medidas biométricas e quanto ao peso ao nascimento, aos 60 e aos 90 dias. Os machos Alpinos foram mais pesados aos 120 dias e apresentaram, ainda, maior ganho de peso no intervalo dos 30 aos 60 dias. O menor custo de produção relativo à alimentação por quilograma de cabrito produzido foi observado aos 90 dias de idade.

Palavras-chave: biometria, crescimento, custo da alimentação, ganho de peso, peso ao nascimento

\section{Performance and body measurements of purebred and crossbred goat kids at different ages}

\begin{abstract}
The objective of this trial was to evaluate performance (weight and weight gain), body measurements, feed conversion, and production costs of purebred (Alpine) and crossbred [1/2 Boer $+1 / 2$ Alpine $(1 / 2$ BA) and 3/4 Boer $+1 / 4$ Alpine (3/4 BA)] goat kids at different ages (60, 90 and 120 days). Therefore, 41 kids in a feedlot system were used in this study. Data were submitted to analysis of variance using the Tukey test for comparison of means. No difference in performance was observed among the three different groups of animals (Alpine, $1 / 2 \mathrm{BA}$, and $3 / 4 \mathrm{BA}$ ). Body condition score was greatest in $3 / 4 \mathrm{BA}$ kids, which may represent additional profit to producers if animals are sold alive. As expected, most body measurements increased with age. Body measurements and weight at birth, 60 and 90 days of age were all greater on males than females. In addition, Alpine males were heavier than females at 120 days of age. They also gained more weight from 30 to 60 days when compared to females. The lowest production cost ( $\mathrm{kg}$ of feed per $\mathrm{kg}$ of weight gain) was observed at 90 days of age.
\end{abstract}

Key Words: birth weight, body measurements, feed cost, growth, weight gain

\section{Introdução}

A população caprina no Brasil é de aproximadamente 10.050.888 de animais, com $94 \%$ concentrada na Região Nordeste (IBGE, 2006). Na Região Sudeste, encontra-se pequena parte do rebanho caprino, mantido em sistema intensivo de criação em pequenas e médias propriedades destinadas à produção leiteira. Nessas propriedades, cabritos machos são geralmente descartados ao nascer por não apresentarem carcaça satisfatória que justifique o alto custo de criação para comercialização. Entretanto, com o aumento da procura por alimentos com características saudáveis, principalmente com baixo teor de gordura, a carne caprina tem ganhado mercado ultimamente. No entanto, poucos estudos têm sido desenvolvidos no intuito de se conhecer a potencialidade desses animais para ganho de peso, desenvolvimento corporal e conversão alimentar para redução dos custos de produção. Também não está bem 
definida a melhor raça de corte para cruzamentos com animais leiteiros visando à produção de cabritos para abate nesse sistema intensivo de criação. Produtores da Região Sudeste, por falta de opção ou modismo, têm utilizado reprodutores da raça Boer para acasalamento com fêmeas do plantel leiteiro ou com animais menos especializados em produção de carne com vistas à complementaridade entre raças e à obtenção de animais mais precoces, com maior rendimento de carcaça e melhor qualidade de carne, capaz de atender às exigências do mercado consumidor. Embora seja válida a iniciativa desses produtores, é preciso maior embasamento científico para comprovação dos resultados desses cruzamentos.

Informações da literatura confirmam a especialidade da raça Boer na produção de carne. Em boas condições de manejo, os animais dessa raça apresentam bom ganho de peso diário (até $200 \mathrm{~g} / \mathrm{dia}$ ) (Van Niekerk \& Casey, 1988), além de boa fertilidade e alta prolificidade (Reynolds, 1985). Tem-se observado que progênies de reprodutores Boer apresentam maior peso ao nascimento em comparação às de outras raças (Waldron et al., 1995; Dhanda et al., 1999; Luo et al., 2000). Tendência semelhante tem sido relatada na literatura também para ganho de peso diário de progênies de reprodutores dessa raça (Goonewardene et al., 1998; Luo et al., 2000; Prieto et al., 2000), sendo registrados ganhos expressivos em cabritos mestiços Boer x Saanen (165 e $140 \mathrm{~g} / \mathrm{dia}$ ) e Saanen x Feral (162 e $140 \mathrm{~g} / \mathrm{dia}$ ). Esses ganhos são superiores aos obtidos em duas épocas de abate nos grupos capretto (14 a $20 \mathrm{~kg}$ ) e chevon (30 a $35 \mathrm{~kg}$ ) (Dhanda et al., 1999).

As raças leiteiras em geral também apresentam elevadas taxas de ganho de peso. Yáñez et al. (2004) observaram em machos Saanen ganhos de 213,3 e 217,8 g/dia nos intervalos de 5 a 20 e de 20 a $35 \mathrm{~kg}$ de PV, respectivamente. Essa elevada taxa de ganho pode, em parte, ser atribuída à intensa seleção dessa raça para precocidade (idade ao primeiro parto) ao longo das gerações (Gonçalves et al., 1997). A idade e o sexo são fatores que também influenciam o ganho de peso. O peso e o ganho de peso nos machos são maiores que em fêmeas (Goonewardene et al., 1998; Mahgoub \& Lodge, 1996). À medida que a idade e/ou o peso corporal aumentam, o ganho de peso decresce (Mourad et al., 1989; Kadim et al., 2003).

As medidas biométricas permitem conhecer o desenvolvimento das diferentes partes que compõem o exterior dos animais e predizer o peso corporal e as características da carcaça. Mohammed \& Amin (1996), em estudo com cabritos Sahel, desenvolveram equações de predição do peso corporal a partir da circunferência do tórax, por consi- derarem a alta correlação entre essas características. Yáñez et al. (2004), em cabritos Saanen, utilizaram medidas biométricas para estimar o peso em jejum, o peso de carcaça fria e a compacidade da carcaça e recomendaram o perímetro torácico e o comprimento corporal, entre outras características, por apresentarem melhor ajuste e facilidade de medição.

Neste estudo, avaliou-se o desempenho de cabritos Alpinos e mestiços Boer $\times$ Alpinos ( $1 / 2 \mathrm{e}$ 3/4 Boer), considerando o peso, o ganho de peso, as medidas biométricas, a conversão alimentar e o custo da alimentação para o aproveitamento desses animais em sistema intensivo de criação para produção de carne.

\section{Material e Métodos}

O experimento foi conduzido na Fazenda Lageado, na área de caprinocultura da Faculdade de Medicina Veterinária e Zootecnia, UNESP, em Botucatu - SP.

Para obtenção dos cabritos, foram utilizadas 30 cabras Alpinas. Metade foi coberta por dois bodes Alpinos e a outra metade por dois bodes Boer, gerando os grupos Alpino (A) e $1 / 2$ Boer $+1 / 2$ Alpina ( $1 / 2 \mathrm{BA}$ ). Também foram utilizadas 15 fêmeas mestiças $(1 / 2$ Boer $+1 / 2$ Alpina) cobertas por bodes Boer para produção dos cabritos do grupo racial 3/4 Boer + $1 / 4$ Alpino (3/4 BA).

Após o nascimento, os cabritos foram separados das mães, receberam tratamento do cordão umbilical e foram pesados, identificados e aleitados com colostro aquecido em banho-maria a $56^{\circ} \mathrm{C}$ durante 60 minutos, duas vezes ao dia, durante três dias, visando evitar a transmissão de CAEV (Artrite Encefalite Caprina Viral). O aleitamento foi feito artificialmente com leite de cabra e de búfala, fornecido em duas refeições diárias até o 70 dia e, a partir da segunda semana, em um único fornecimento diário, sempre pela manhã. A quantidade de leite oferecida diariamente não ultrapassou 1,5 L e o desmame foi realizado aos 60 dias.

A partir da segunda e até a quarta semana de idade, os cabritos tiveram à disposição água limpa e fresca, além de concentrado farelado e péletes com $70 \%$ de concentrado e $30 \%$ de feno de coastcross. A partir da quarta semana (28 dias), foram alojados em nove baias coletivas, segundo o grupo racial e a idade de abate presumida, onde receberam somente a dieta peletizada.

O concentrado utilizado foi composto de $49 \%$ de milho, $38 \%$ de farelo de soja, $10 \%$ de farelo de algodão, $2 \%$ de calcário e $1 \%$ de sal mineral. A dieta completa $(90,33 \%$ de MS, $13,85 \%$ de $\mathrm{PB}, 4,54 \%$ de EE, $9,35 \%$ de minerais, $13,50 \%$ de FB, 27,79\% de FDN e 15,10\% de FDA) foi calculada para atender às exigências de mantença e para possibilitar ganho 
de $150 \mathrm{~g} / \mathrm{dia}$ (NRC, 1981). O fornecimento da dieta sólida foi feito pela manhã. As sobras foram pesadas para ajuste da quantidade fornecida diariamente visando manter em torno de 10 a $15 \%$ de sobras. Semanalmente, foi avaliada a ingestão média de alimentos por baia para avaliação do consumo e da conversão alimentar por grupo racial.

Foram utilizados 41 animais (16 Alpinos, 14 1/2 Boer $\times$ Alpino e 11 3/4 Alpino). Os animais foram incluídos no experimento aos 32,85 dias de idade, em média (P30 - 24 a 34 dias de idade), quando estavam recebendo somente a dieta peletizada. Durante o experimento, foram avaliados aos 68,17 dias (P60 - 59 a 79 dias de idade), aos 96,55 dias (P90 - 87 a 107 dias de idade) e aos 124,81 dias (P120 - 118 a 134 dias de idade). Aos 60, 90 e aos 120 dias, após avaliação, um terço de cada grupo racial foi abatido para determinação das características de carcaça. Nas Tabelas 2 e 3 consta o número de animais avaliados em cada fase.

Os animais foram pesados semanalmente pela manhã, antes do fornecimento da água e da dieta sólida, para avaliação do peso vivo e do ganho de peso ao nascer, aos 30, 60, 90 e 120 dias de idade. Os dados de peso e ganho de peso foram avaliados por análise de variância e as médias comparadas pelo teste Tukey $(\mathrm{P}<0,05)$. Na análise das características de desempenho, foram considerados o grupo racial, o sexo, a interação grupo racial $\times$ sexo e a idade no dia da avaliação como covariável.

Ao atingirem as idades de avaliação, os animais foram colocados sobre uma superfície plana para determinação da condição corporal e das medidas biométricas. A condição corporal foi determinada pela atribuição de escore, utilizando-se escala de 0 a 5 pontos $(0=$ animal extremamente magro e 5 = animal gordo), e mediante visualização e palpação da coluna vertebral logo após o 13o par de costelas (Mourad et al., 1989).

As medidas biométricas foram obtidas utilizando-se uma fita métrica e uma régua de madeira. Foram tomadas as seguintes medidas: comprimento corporal - distância entre a articulação cervicotorácica e a base da cauda; altura anterior - distância entre a região da cernelha e a extremidade distal do membro anterior; altura posterior distância entre a tuberosidade sacral, na garupa, e a extremidade distal do membro posterior; perímetro torácico perímetro tomando-se como base o esterno e a cernelha, passando por trás da paleta; perímetro da perna - perímetro tomando-se como base a parte média da perna, acima da articulação femurotibiopatelar; largura da garupa distância entre os trocânteres maiores dos fêmures; largura do peito - distância entre as faces das articulações escápuloumerais.
Utilizou-se análise de variância para avaliação das medidas biométricas e aplicou-se o teste Tukey $(\mathrm{P}<0,05)$ para comparação das médias. Foram considerados os efeitos de idade de avaliação, grupo racial, sexo e a interação idade de avaliação $\times$ grupo racial. Para os dois modelos, foi utilizado o programa SAEG, versão 7.0 (UFV, 1997).

$O$ custo da alimentação por fase de crescimento foi estimado com base no consumo médio de leite e de dieta sólida por animal multiplicado por $\mathrm{R} \$ 0,60$ e $\mathrm{R} \$ 0,434$, respectivamente, valores praticados para o leite bovino e para o custo da dieta, considerando a média dos preços dos ingredientes comercializados na região em fevereiro de 2005. O custo acumulado foi obtido pela soma do custo de cada fase, enquanto o custo por quilo de cabrito foi calculado pela divisão do custo acumulado pelo ganho de peso acumulado em cada fase.

\section{Resultados e Discussão}

Os pesos aos 60, 90 e aos 120 dias foram de 14,63 $\pm 0,34$,

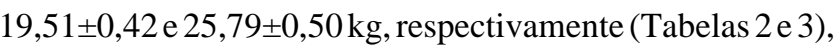
e podem ser considerados satisfatórios para caprinos, pois foram superiores aos encontrados na literatura com animais mestiços Boer (Goonewardene et al., 1998; Dhanda et al., 1999) e de outras raças (Johnson et al., 1995).

Não foi observada diferença entre grupos raciais para o peso ao nascimento (Tabela 1). Este resultado, no entanto, diverge dos registrados na literatura (Goonewardene et al., 1998; Dhanda et al., 1999; Luo et al., 2000), que descrevem maior peso ao nascer para animais filhos de reprodutores Boer.

Os pesos aos 30, 60 e aos 90 dias de idade também não diferiram entre grupos raciais (Tabela 1), possivelmente porque ambas as raças são de porte elevado e de alto potencial de crescimento em sistema de confinamento. De modo geral, esse resultado foi semelhante aos encontrados por Goonewardene et al. (1998) e Dhanda et al. (1999), que não observaram diferença entre animais resultantes do cruzamento de Boer com raças leiteiras (Alpina e Saanen)e animais de raças puras leiteiras. Contudo, esses autores notaram diferença quando compararam esses cruzamentos (Boer $\times$ leiteiros) a animais mestiços de Boer com raças nativas e nativas puras, possivelmente em virtude do menor porte das raças nativas.

As raças leiteiras têm sido intensamente selecionadas para idade ao primeiro parto (IPP), característica que possui 0,25 e 0,50 de herdabilidade (Singh et al., 1970; Gonçalves et al., 1997), valor considerado elevado e que indica altos ganhos por geração. A seleção para idade ao primeiro parto 
Tabela 1 - Resumo da análise de variância das características de desempenho

Table 1 - Summary of analysis of variance for performance traits

\begin{tabular}{|c|c|c|c|c|c|}
\hline \multirow[t]{2}{*}{$\begin{array}{l}\text { Característica } \\
\text { Trait }\end{array}$} & \multicolumn{5}{|c|}{$\begin{array}{l}\text { Fonte de variação } \\
\text { Source of variation }\end{array}$} \\
\hline & $\begin{array}{c}\text { Grupo racial }(\mathrm{GL}) \\
\text { Breed }(D F)\end{array}$ & $\begin{array}{l}\text { Sexo }(\mathrm{GL}) \\
\text { Gender }(D F)\end{array}$ & $\begin{array}{c}\text { Grupo racial } \mathrm{x} \text { sexo (GL) } \\
\text { Breed } x \text { gender }(D F)\end{array}$ & $\begin{array}{c}\text { Idade: linear }(\mathrm{GL}) \\
\text { Age: linear }(D F)\end{array}$ & $\begin{array}{l}\text { Resíduo (GL) } \\
\quad \text { Error }(D F)\end{array}$ \\
\hline \multicolumn{6}{|l|}{ Quadrado médio (Mean square) } \\
\hline Peso ao nascer (Birth weight) & $0,48(2)$ & $1,00 *(1)$ & $0,13(2)$ & & $0,19(35)$ \\
\hline Peso aos 30 dias (Weight at 30 days) & $0,54(2)$ & $2,68(1)$ & $1,69(2)$ & $10,97 *(1)$ & $1,27(34)$ \\
\hline Peso aos 60 dias (Weight at 60 days) & $0,20(2)$ & $45,15 *(1)$ & $6,56(2)$ & $21,22 *(1)$ & $4,68(34)$ \\
\hline Peso aos 90 dias (Weight at 90 days) & $1,44(2)$ & $59,75 *(1)$ & $11,95(2)$ & $17,53 *(1)$ & $4,99(22)$ \\
\hline Peso aos 120 dias (Weight at 120 days) & $0,27(2)$ & $75,66 *(1)$ & $16,46 *(2)$ & $15,92 *(1)$ & $3,78(9)$ \\
\hline
\end{tabular}

${ }^{*} \mathrm{P}<0,05(P<0.05), \mathrm{GL}-$ graus de liberdade $(D F-$ Degrees of freedom).

Tabela 2 - Médias de peso vivo $(\mathrm{kg})$ de cabritos de diferentes grupos raciais

Table 2 - Body weight of purebred and crossbred kids at different ages

\begin{tabular}{|c|c|c|c|c|c|c|}
\hline \multirow[t]{2}{*}{$\begin{array}{l}\text { Característica (kg) } \\
\text { Trait }\end{array}$} & \multirow[t]{2}{*}{$\begin{array}{l}\text { Média } \\
\text { Mean }\end{array}$} & \multicolumn{3}{|c|}{$\begin{array}{c}\text { Grupo racial } \\
\text { Breed }\end{array}$} & \multicolumn{2}{|c|}{$\begin{array}{c}\text { Sexo } \\
\text { Gender }\end{array}$} \\
\hline & & $\begin{array}{l}\text { Alpino } \\
\text { Alpine }\end{array}$ & $1 / 2$ Boer & $3 / 4$ Boer & $\begin{array}{l}\text { Macho } \\
\text { Male }\end{array}$ & $\begin{array}{l}\text { Fêmea } \\
\text { Female }\end{array}$ \\
\hline $\begin{array}{l}\text { Peso ao nascer } \\
\text { Birth weight }\end{array}$ & $\begin{array}{c}3,68 \pm 0,07 \\
(41)\end{array}$ & $\begin{array}{c}3,61 \pm 0,11 \\
(16)\end{array}$ & $\begin{array}{c}3,90 \pm 0,11 \\
(14)\end{array}$ & $\begin{array}{c}3,54 \pm 0,13 \\
(11)\end{array}$ & $\begin{array}{c}3,84 \pm 0,09 a \\
(24)\end{array}$ & $\begin{array}{c}3,52 \pm 0,10 b \\
(17)\end{array}$ \\
\hline Peso aos 30 dias & $7,46 \pm 0,18$ & $7,35 \pm 0,31$ & $7,69 \pm 0,31$ & $7,33 \pm 0,35$ & $7,72 \pm 0,23$ & $7,19 \pm 0,28$ \\
\hline $\begin{array}{l}\text { Weight at } 30 \text { days } \\
\text { Peso aos } 60 \text { dias }\end{array}$ & $\begin{array}{c}(41) \\
14,63 \pm 0,34\end{array}$ & $\begin{array}{c}(16) \\
14,69 \pm 0,59\end{array}$ & $\begin{array}{c}(14) \\
14,73 \pm 0,59\end{array}$ & $\begin{array}{c}(11) \\
14,48 \pm 0,67\end{array}$ & $\begin{array}{c}(24) \\
15,73 \pm 0,45 \mathrm{a}\end{array}$ & $\begin{array}{c}(17) \\
13,53 \pm 0,54 b\end{array}$ \\
\hline Weight at 60 days & (41) & (16) & (14) & (11) & (24) & (17) \\
\hline $\begin{array}{l}\text { Peso aos } 90 \text { dias } \\
\text { Weight at } 90 \text { days }\end{array}$ & $\begin{array}{c}19,51 \pm 0,42 \\
(29)\end{array}$ & $\begin{array}{c}19,11 \pm 0,72 \\
(11)\end{array}$ & $\begin{array}{c}19,90 \pm 0,71 \\
(10)\end{array}$ & $\begin{array}{c}19,50 \pm 0,80 \\
(8)\end{array}$ & $\begin{array}{c}20,99 \pm 0,57 \mathrm{a} \\
(16)\end{array}$ & $\begin{array}{c}18,02 \pm 0,63 b \\
(13)\end{array}$ \\
\hline
\end{tabular}

Para cada fonte de variação, médias seguidas de mesma letra não diferem $(\mathrm{P}<0,05)$ pelo teste Tukey (For each variation source, means followed by the same letter do not differ $(P<0.05)$ by Tukey test $)$

( ) Número de animais [( ) Number of animals].

reflete no ganho de peso durante a fase de crescimento. Por isso, embora a raça Boer proporcione elevados ganhos de peso, não supera as raças leiteiras, que passaram por longos períodos de seleção.

Os machos foram mais pesados que as fêmeas ao nascer, aos 60 e aos 90 dias (Tabela 2). No grupo Alpino, os machos foram mais pesados também aos 120 dias, comprovando crescimento muito diferenciado dos machos em relação às fêmeas dessa raça (Tabela 3), indicando que a diferença entre sexos em animais mestiços tende a desaparecer com o aumento da idade, o que não ocorre em animais Alpinos. Diferença nos pesos entre sexos também foi observada por Goonewardene et al. (1998), em caprinos com idades semelhantes à dos animais utilizados neste trabalho.

O resumo da análise de variância para o ganho de peso é apresentada na Tabela 4 e as médias em função das fontes de variação estudadas, na Tabela 5. Não houve influência do grupo racial e do sexo no ganho de peso em nenhum dos intervalos estudados, exceto no intervalo GP3060, no qual os machos apresentaram maior ganho de peso. Os resultados encontrados para o efeito do grupo racial no ganho de peso diferem do relatado por outros autores (Dhanda et al., 1999; Luo et al., 2000; Prieto et al., 2000), o que permite inferir sobre as mesmas considerações feitas para peso; ou seja, os mestiços de Boer com raças leiteiras não diferem dos animais de origem leiteira, principalmente em sistemas de confinamento, nos quais a alimentação atende às exigências nutricionais e não permite aos animais externar diferenças na capacidade de tolerar as adversidades do ambiente nem a habilidade de procurar alimentos.

O ganho de peso obtido neste trabalho, nas diferentes idades de avaliação, foi elevado se comparado ao encontrado em outros trabalhos com mestiços Boer. Dhanda et al. (1999) observaram ganhos de peso de 128 a $165 \mathrm{~g}$ /dia do nascimento aos 101 dias em cinco grupos raciais de cabritos criados em pastagem e abatidos ao desmame com 14 a $20 \mathrm{~kg}$ de PV. Em sistema de confinamento, Luo et al. (2000) constataram em três grupos raciais ganhos de peso de 60,3 a 76,8 g/dia do nascimento ao desmame (oito semanas), enquanto Goonewardene et al. (1998), em seis grupos raciais (três 
Tabela 3 - Efeito da interação sexo $\times$ grupo racial sobre o peso aos 120 dias de idade (P120)

Table 3 - Effect of the gender $\times$ breed interaction on body weight at 120 days of age

\begin{tabular}{|c|c|c|c|c|c|}
\hline \multirow[t]{2}{*}{$\begin{array}{l}\text { Característica }(\mathrm{kg}) \\
\text { Trait }\end{array}$} & \multirow[t]{2}{*}{$\begin{array}{l}\text { Média } \\
\text { Mean }\end{array}$} & \multirow[t]{2}{*}{$\begin{array}{l}\text { Sexo } \\
\text { Gender }\end{array}$} & \multicolumn{3}{|c|}{$\begin{array}{c}\text { Grupo racial } \\
\text { Breed }\end{array}$} \\
\hline & & & $\begin{array}{l}\text { Alpino } \\
\text { Alpine }\end{array}$ & $1 / 2$ Boer & 3/4 Boer \\
\hline \multirow[t]{2}{*}{$\begin{array}{l}\text { Peso aos } 120 \text { dias }(\mathrm{kg}) \\
\text { Weight at } 120 \text { days }\end{array}$} & $\begin{array}{l}25,79 \pm 0,50 \\
(16)\end{array}$ & $\begin{array}{l}\text { Macho } \\
\text { Male }\end{array}$ & $\begin{array}{c}29,98 \pm 1,09 \mathrm{Aa} \\
(4)\end{array}$ & $\begin{array}{c}27,13 \pm 1,39 \mathrm{Aa} \\
(2)\end{array}$ & $\begin{array}{c}27,08 \pm 1,39 \mathrm{Aa} \\
(2)\end{array}$ \\
\hline & & $\begin{array}{l}\text { Fêmea } \\
\text { Female }\end{array}$ & $\begin{array}{c}21,12 \pm 1,15 \mathrm{Ab} \\
(3)\end{array}$ & $\begin{array}{c}24,59 \pm 1,20 \mathrm{Aa} \\
(3)\end{array}$ & $\begin{array}{c}24,88 \pm 1,46 \mathrm{Aa} \\
(2)\end{array}$ \\
\hline
\end{tabular}

Para sexo, médias seguidas de mesma letra maiúscula na linha não diferem pelo teste Tukey (For each sex, means followed by same capital letter in a row do not differ). Para grupo racial, médias seguidas de mesma letra minúscula na coluna não diferem $(\mathrm{P}<0,05)$ pelo teste Tukey (For each breed, means followed by the same small letter in a column, do not differ $(P<0.05)$ by Tukey test).

( ) Número de animais [( ) Number of animals].

Tabela 4 - Análise de variância das características de ganho de peso

Table 4 - Summary of analysis of variance for weight gain at different ages

\begin{tabular}{|c|c|c|c|c|}
\hline \multirow[t]{2}{*}{$\begin{array}{l}\text { Característica } \\
\text { Trait }\end{array}$} & \multicolumn{4}{|c|}{$\begin{array}{l}\text { Fonte de variação } \\
\text { Source of variation }\end{array}$} \\
\hline & $\begin{array}{c}\text { Grupo racial } \\
\text { (GL) } \\
\text { Breed (DF) }\end{array}$ & $\begin{array}{c}\text { Sexo } \\
(\mathrm{GL}) \\
\text { Gender }(D F)\end{array}$ & $\begin{array}{c}\text { Grupo racial } \mathrm{x} \text { sexo } \\
(\mathrm{GL}) \\
\text { Breed } x \text { gender }(D F)\end{array}$ & $\begin{array}{l}\text { Resíduo } \\
\quad(\mathrm{GL}) \\
\text { Error }(D F)\end{array}$ \\
\hline & \multicolumn{4}{|c|}{$\begin{array}{l}\text { Quadrado médio } \\
\text { Mean square }\end{array}$} \\
\hline Ganho de peso do nascimento aos 30 dias & 22 & 432 & 908 & 962 \\
\hline Weight gain from birth to 30 days & (2) & (1) & (2) & (35) \\
\hline Ganho de peso dos 30 aos 60 dias & 849 & $23.889 *$ & 22 & 1.967 \\
\hline Weight gain from 30 to 60 days & (2) & (1) & (2) & (35) \\
\hline Ganho de peso dos 60 aos 90 dias & 505 & 14.309 & 2.347 & 4.486 \\
\hline Gain weight from 60 to 90 days & (2) & (1) & (2) & (35) \\
\hline Ganho de peso dos 90 aos 120 dias & 2.869 & 7.597 & 1.437 & 2.206 \\
\hline Weight gain from 90 to 120 days & (2) & (1) & (2) & (35) \\
\hline
\end{tabular}

${ }^{*} \mathrm{P}<0,05(P<0.05), \mathrm{GL}-$ graus de liberdade $(D F-$ Degrees of freedom).

Tabela 5 - Características de ganho de peso de caprinos de diferentes grupos raciais

Table 5 - Body weight gain of purebred and crossbred kids at different ages

\begin{tabular}{|c|c|c|c|c|c|c|}
\hline \multirow[t]{2}{*}{$\begin{array}{l}\text { Característica }(\mathrm{kg}) \\
\text { Trait }\end{array}$} & \multirow[t]{2}{*}{$\begin{array}{l}\text { Média } \\
\text { Mean }\end{array}$} & \multicolumn{3}{|c|}{$\begin{array}{l}\text { Grupo racial } \\
\text { Breed }\end{array}$} & \multicolumn{2}{|c|}{$\begin{array}{l}\text { Sexo } \\
\text { Gender }\end{array}$} \\
\hline & & $\begin{array}{l}\text { Alpino } \\
\text { Alpine }\end{array}$ & $1 / 2$ Boer & $3 / 4$ Boer & $\begin{array}{l}\text { Macho } \\
\text { Male }\end{array}$ & $\begin{array}{l}\text { Fêmea } \\
\text { Female }\end{array}$ \\
\hline $\begin{array}{l}\text { Ganho de peso do nascimento aos } 30 \text { dias } \\
\text { Weight gain from birth to } 30 \text { days }\end{array}$ & $113,7 \pm 4,9$ & $114,3 \pm 8,0$ & $114,7 \pm 8,3$ & $112,1 \pm 9,3$ & $117,1 \pm 6,4$ & $110,4 \pm 7,5$ \\
\hline $\begin{array}{l}\text { Ganho de peso dos } 30 \text { aos } 60 \text { dias } \\
\text { Weight gain } 30 \text { to } 60 \text { days }\end{array}$ & $202,6 \pm 7,1$ & $211,3 \pm 11,4$ & $196,6 \pm 11,9$ & $199,8 \pm 13,4$ & $227,4 \pm 9,2 \mathrm{a}$ & $177,8 \pm 10,8 b$ \\
\hline $\begin{array}{l}\text { Ganho de peso dos } 60 \text { aos } 90 \text { dias } \\
\text { Gain weight from } 60 \text { to } 90 \text { days }\end{array}$ & $179,5 \pm 12,6$ & $172,4 \pm 20,9$ & $186,5 \pm 21,1$ & $179,6 \pm 23,6$ & $202,2 \pm 17,2$ & $156,9 \pm 18,7$ \\
\hline $\begin{array}{l}\text { Ganho de peso dos } 90 \text { aos } 120 \text { dias } \\
\text { Weight gain from } 90 \text { to } 120 \text { days }\end{array}$ & $201,4 \pm 12,1$ & $194,6 \pm 17,9$ & $230,2 \pm 21,4$ & $182,4 \pm 23,4$ & $225,0 \pm 17,5$ & $179,8 \pm 16,9$ \\
\hline
\end{tabular}

Para cada fonte de variação, médias seguidas de mesma letra não diferem $(\mathrm{P}<0,05)$ pelo teste Tukey (For each variation source, means followed by the same letter do not differ $(P<0.05)$ by Tukey test).

provenientes de reprodutores Boer e três de Alpinos), registraram ganhos de 119 a 161 do nascimento ao desmame ( $90 \pm 13$ dias), de 130 a 181 do nascimento aos 100 dias e de 87 a $124 \mathrm{~g} /$ dia do desmame aos 160 dias. Esse maior ganho pode ter sido conseqüência da forma de apresentação da dieta (peletizada), que pode ter estimulado o consumo de MS e a utilização de grupos raciais constituídos de animais das raças Boer e Alpina, reconhecidas por suas habilidades de ganho de peso (Van Niekerk \& Casey, 1988; Yáñez et al., 2004; Gonçalves et al., 1997). 
Observa-se na Tabela 5 que houve redução no ganho de peso no intervalo de 60 a 90 dias e recuperação no intervalo seguinte, possivelmente em razão do estresse provocado pelo desaleitamento realizado aos 60 dias e da adaptação à dieta sólida.

A conversão alimentar ( $\mathrm{kg}$ de alimento / $\mathrm{kg}$ de ganho) e a relação ganho de peso (g) / quilo de alimento consumido (GP/AL) obtidas nos diferentes grupos raciais e nas três idades de avaliação encontram-se na Tabela 6.

A relação entre ganho de peso $(\mathrm{g})$ por quilo de alimento consumido maior que $1.000 \mathrm{~g}$ no período de $30 \mathrm{a} 60$ dias foi ocasionada pelo fato de que, nessa idade, os animais ainda estavam recebendo $1,5 \mathrm{~L}$ de leite. Verificou-se aumento na conversão alimentar com o avançar da idade. A mais eficiente taxa de conversão ocorreu no intervalo de 30 a 60 dias e possivelmente resultou do baixo consumo de alimento sólido decorrente do aleitamento.

Na Tabela 7 são apresentados os custos relativos à alimentação (por idade de avaliação) considerando os pesos médios (Tabela 2) e a conversão alimentar (Tabela 6). O custo final da alimentação para a produção de $25,79 \mathrm{~kg}$ de cabrito foi de $\mathrm{R} \$ 85,61$, ou $\mathrm{R} \$ 3,31$ por quilo vivo de cabrito produzido. Considerando que o custo com alimentação representa $50 \%$ do custo total, o custo final aumentaria para $\mathrm{R} \$ 6,62$ e o custo por quilo de carcaça para $\mathrm{R} \$ 13,24$ considerando um rendimento de carcaça de $50 \%$.

Desconsiderando o custo de $\mathrm{R} \$ 54,00$ com o fornecimento de leite (o produtor muitas vezes tem excedente desse alimento por não conseguir comercializar), o custo com alimentação seria de $\mathrm{R} \$ 31,61$ e o custo por quilo vivo de cabrito produzido seria de $\mathrm{R} \$ 1,22$. Se fossem mantidos constantes o percentual da alimentação no custo total (50\%) e o rendimento de carcaça (50\%), o custo final do quilo de carcaça seria de $R \$ 4,88$ ou $R \$ 73,20$ por arroba de cabrito e, uma vez que o preço médio de venda da carcaça de cabrito na Região Sudeste é de três vezes a arroba do boi $(\mathrm{R} \$ 150,00)$, a obtenção de lucro ficaria condicionada à redução do custo com a alimentação líquida.

O menor custo $/ \mathrm{kg}$ de peso vivo de cabrito produzido foi de $\mathrm{R} \$ 3,25$ aos 90 dias, comprovando que essa é a idade recomendada para obtenção de maior lucro, apesar do elevado custo de produção.

Na Tabela 8 constam os resumos das análises de variância das medidas biométricas e, na Tabela 9, suas médias de acordo

Tabela 6 - Conversão alimentar e relação ganho de peso por quilo $(\mathrm{g} / \mathrm{kg})$ de alimento sólido consumido nos diferentes grupos raciais em três idades de avaliação

Table 6 - Feed conversion and feed efficiency $(\mathrm{g} / \mathrm{kg})$ of purebred and crossbred kids at different ages

\begin{tabular}{|c|c|c|c|c|}
\hline \multirow[t]{2}{*}{$\begin{array}{l}\text { Característica } \\
\text { Trait }\end{array}$} & \multirow[t]{2}{*}{$\begin{array}{l}\text { Média } \\
\text { Mean }\end{array}$} & \multicolumn{3}{|c|}{$\begin{array}{l}\text { Grupo racial } \\
\text { Breed }\end{array}$} \\
\hline & & $\begin{array}{l}\text { Alpino } \\
\text { Alpine }\end{array}$ & $1 / 2$ Boer & $3 / 4$ Boer \\
\hline $\begin{array}{l}\text { Ganho de peso do nascimento aos } 30 \text { dias } \\
\text { Weight gain from birth to } 30 \text { days }\end{array}$ & $0,892(1121)$ & $0,959(1043)$ & $0,865(1156)$ & $0,818 \quad(1222)$ \\
\hline $\begin{array}{l}\text { Ganho de peso dos } 30 \text { aos } 60 \text { dias } \\
\text { Weight gain from } 30 \text { to } 60 \text { days }\end{array}$ & $3,156(316)$ & $3,300(303)$ & $3,096 \quad(323)$ & $3,048 \quad(328)$ \\
\hline Ganho de peso dos 60 aos 90 dias & $8,132(123)$ & 7,735 (129) & $8,606(116)$ & $8,123(123)$ \\
\hline
\end{tabular}

( ) relação entre ganho de peso por quilo de alimento sólido consumido $(\mathrm{g} / \mathrm{kg})$.

( ) feed efficiency $(\mathrm{g} / \mathrm{kg})$.

Tabela 7 - Custo com alimentação em cada idade de avaliação

Table 7 - $\quad$ Feed costs of purebred and crossbred kids at different ages

\begin{tabular}{|c|c|c|c|c|c|c|}
\hline $\begin{array}{l}\text { Idade de avaliação } \\
\text { Age }\end{array}$ & $\begin{array}{l}\text { Ganho de peso }(\mathrm{kg}) \\
\text { Weight gain }(\mathrm{kg})\end{array}$ & $\begin{array}{l}\text { Leite }(\mathrm{L}) \\
\operatorname{Milk}(L)\end{array}$ & $\begin{array}{l}\text { CAS }(\mathrm{kg}) \\
C S F(k g)\end{array}$ & $\begin{array}{c}\text { Custo/fase }(\mathrm{R} \$) \\
\text { Cost/Phase }(R \$)\end{array}$ & $\begin{array}{r}\mathrm{CA} / \mathrm{F}(\mathrm{R} \$) \\
O C / P(R \$)\end{array}$ & $\begin{array}{c}\mathrm{C} / \mathrm{kg} \mathrm{PV}(\mathrm{R} \$) \\
C / \mathrm{kg} B W(R \$)\end{array}$ \\
\hline $\begin{array}{l}\text { Nascimento aos } 30 \text { dias } \\
\text { From birth to } 30 \text { days }\end{array}$ & $3,78(7,46-3,68)$ & 45 & - & 27,00 & 27 & 3,62 \\
\hline 30 aos 60 dias & $7,17(14,63-7,46)$ & 45 & & 27,00 & & \\
\hline From 30 to 60 days & & & 6,39 & 2,77 & 56,77 & 3,88 \\
\hline $\begin{array}{l}60 \text { aos } 90 \text { dias } \\
\text { From } 60 \text { to } 90 \text { days }\end{array}$ & $4,88(19,51-14,63)$ & & 15,40 & 6,68 & 63,45 & 3,25 \\
\hline $\begin{array}{l}90 \text { aos } 120 \text { dias } \\
\text { From } 90 \text { to } 120 \text { days }\end{array}$ & $6,28(25,79-19,51)$ & & 51,07 & 22,16 & 85,61 & 3,31 \\
\hline
\end{tabular}

CAS: consumo de alimento sólido, C/F: custo por fase, CA/F: custo acumulado por fase, C/kg PV: custo por kg de peso vivo. CSF: solid feed intake, C/F: cost per phase, AC/P: overall cost/phase, C/kg LW: cost per kg of body weight. 
Tabela 8 - Resumo da análise de variância do escore corporal e das medidas biométricas Table 8 - Summary of analysis of variance for body score and body measurements

\begin{tabular}{|c|c|c|c|c|c|}
\hline \multirow[t]{2}{*}{$\begin{array}{l}\text { Característica } \\
\text { Trait }\end{array}$} & \multicolumn{5}{|c|}{$\begin{array}{l}\text { Fonte de variação } \\
\text { Source of variation }\end{array}$} \\
\hline & $\begin{array}{c}\text { Idade de avaliação } \\
(\mathrm{GL}) \\
\text { Age }(D F)\end{array}$ & $\begin{array}{l}\text { Grupo racial } \\
(\mathrm{GL}) \\
\text { Breed }(D F)\end{array}$ & $\begin{array}{c}\text { Sexo } \\
(\mathrm{GL}) \\
\text { Gender }(D F)\end{array}$ & $\begin{array}{c}\text { Idade de avaliação x Grupo racial } \\
(\mathrm{GL}) \\
\text { Age } \times \text { Breed }(D F)\end{array}$ & $\begin{array}{l}\text { Resíduo } \\
\quad(\mathrm{GL}) \\
\text { Error }(D F)\end{array}$ \\
\hline \multicolumn{6}{|c|}{$\begin{array}{l}\text { Quadrado médio } \\
\text { Mean square }\end{array}$} \\
\hline Escore corporal & $1,83^{*}$ & $0,79 *$ & 0,16 & 0,13 & 0,09 \\
\hline Body score & (2) & (2) & (1) & (4) & (31) \\
\hline Comprimento corporal & $414,13 *$ & 32,01 & $96,80 *$ & 9,71 & 13,40 \\
\hline Body length & (2) & (2) & (1) & (4) & (31) \\
\hline Altura anterior & $253,73 *$ & $75,09 *$ & $99,93 *$ & 9,86 & 8,16 \\
\hline Fore height & (2) & (2) & (1) & (4) & (31) \\
\hline Altura posterior & $247,48 *$ & $45,64 *$ & $75,18 *$ & 9,48 & 7,48 \\
\hline Body height & (2) & (2) & (1) & (4) & (31) \\
\hline Perímetro torácico & $482,71 *$ & 9,98 & $126,30 *$ & 5,42 & 8,03 \\
\hline Thoracic perimeter & (2) & (2) & (1) & (4) & (31) \\
\hline Perímetro da perna & $206,36^{*}$ & 0,67 & $36,72 *$ & 3,21 & 6,63 \\
\hline Leg perimeter & (2) & (2) & (1) & (4) & $(31)$ \\
\hline Largura da garupa & $17,84^{*}$ & 0,48 & $4,77^{*}$ & 2,39 & 0,98 \\
\hline Rump width & (2) & (2) & (1) & (4) & (31) \\
\hline Largura do peito & $9,00 *$ & 0,16 & $22,08 *$ & 0,72 & 1,06 \\
\hline Chest width & (2) & (2) & (1) & (4) & (31) \\
\hline
\end{tabular}

${ }^{*} \mathrm{P}<0,05(P<0.05), \mathrm{GL}-$ graus de liberdade ( $D F-$ Degrees of freedom) .

Tabela 9 - Escore corporal e medidas biométricas de cabritos puros e mestiços em diferentes idades Table 9 - Body score and body measurements of purebred and crossbred kids at different ages

\begin{tabular}{|c|c|c|c|c|c|c|c|c|c|}
\hline \multirow[t]{2}{*}{$\begin{array}{l}\text { Característica } \\
\text { Trait }\end{array}$} & \multirow[t]{2}{*}{$\begin{array}{l}\text { Média } \\
\text { Mean }\end{array}$} & \multicolumn{3}{|c|}{$\begin{array}{c}\text { Idade de avaliação } \\
\text { Age }\end{array}$} & \multicolumn{3}{|c|}{$\begin{array}{c}\text { Grupo racial } \\
\text { Breed }\end{array}$} & \multicolumn{2}{|c|}{$\begin{array}{l}\text { Sexo } \\
\text { Gender }\end{array}$} \\
\hline & & 60 & 90 & 120 & $\begin{array}{l}\text { Alpino } \\
\text { Alpine }\end{array}$ & $1 / 2$ Boer & $3 / 4$ Boer & $\begin{array}{l}\text { Macho } \\
\text { Male }\end{array}$ & $\begin{array}{l}\text { Fêmea } \\
\text { Female }\end{array}$ \\
\hline $\begin{array}{l}\mathrm{EC}(0-5) \\
B S(0-5)\end{array}$ & $2,7 \pm 0,1$ & $3,1 \pm 0,1 \mathrm{a}$ & $2,3 \pm 0,1 \mathrm{c}$ & $2,7 \pm 0,1 \mathrm{~b}$ & $2,4 \pm 0,1 \mathrm{~b}$ & $2,7 \pm 0,1 \mathrm{ab}$ & $2,9 \pm 0,1 \mathrm{a}$ & $2,8 \pm 0,1$ & $2,6 \pm 0,1$ \\
\hline $\begin{array}{l}C C(\mathrm{~cm}) \\
B L(\mathrm{~cm})\end{array}$ & $67,1 \pm 0,6$ & $61,3 \pm 1,2 \mathrm{c}$ & $66,9 \pm 1,0 \mathrm{~b}$ & $73,1 \pm 0,9 \mathrm{a}$ & $68,7 \pm 0,9$ & $67,0 \pm 1,0$ & $65,6 \pm 1,1$ & $68,8 \pm 0,7 \mathrm{a}$ & $65,5 \pm 0,9 b$ \\
\hline $\begin{array}{l}\text { AA }(\mathrm{cm}) \\
F H(\mathrm{~cm})\end{array}$ & $55,3 \pm 0,5$ & $50,9 \pm 0,9 \mathrm{c}$ & $55,1 \pm 0,8 \mathrm{~b}$ & $60,1 \pm 0,7 \mathrm{a}$ & $57,9 \pm 0,7 \mathrm{a}$ & $54,9 \pm 0,8 \mathrm{~b}$ & $53,2 \pm 0,9 b$ & $57,0 \pm 0,6 \mathrm{a}$ & $53,7 \pm 0,7 b$ \\
\hline $\begin{array}{l}\text { AP }(\mathrm{cm}) \\
H H(\mathrm{~cm})\end{array}$ & $55,5 \pm 0,4$ & $50,9 \pm 0,9 \mathrm{c}$ & $55,4 \pm 0,7 \mathrm{~b}$ & $60,1 \pm 0,7 \mathrm{a}$ & $57,5 \pm 0,7 \mathrm{a}$ & $55,2 \pm 0,8 \mathrm{ab}$ & $53,7 \pm 0,8 b$ & $56,9 \pm 0,6 \mathrm{a}$ & $54,0 \pm 0,7 b$ \\
\hline $\begin{array}{l}\mathrm{PT}(\mathrm{cm}) \\
T P(\mathrm{~cm})\end{array}$ & $61,3 \pm 0,5$ & $55,5 \pm 0,9 \mathrm{c}$ & $60,3 \pm 0,8 \mathrm{~b}$ & $67,9 \pm 0,7 \mathrm{a}$ & $62,2 \pm 0,7$ & $61,0 \pm 0,8$ & $60,5 \pm 0,9$ & $63,1 \pm 0,6 a$ & $59,4 \pm 0,7 b$ \\
\hline $\begin{array}{l}P P(\mathrm{~cm}) \\
L P(\mathrm{~cm})\end{array}$ & $29,7 \pm 0,4$ & $25,7 \pm 0,8 \mathrm{c}$ & $29,4 \pm 0,7 b$ & $33,9 \pm 0,7 \mathrm{a}$ & $29,8 \pm 0,7$ & $29,8 \pm 0,7$ & $29,4 \pm 0,8$ & $30,7 \pm 0,5 \mathrm{a}$ & $28,7 \pm 0,7 b$ \\
\hline $\begin{array}{l}\mathrm{LG}(\mathrm{cm}) \\
R W(\mathrm{~cm})\end{array}$ & $25,0 \pm 0,2$ & $23,7 \pm 0,3 b$ & $25,2 \pm 0,3 \mathrm{a}$ & $26,1 \pm 0,3 \mathrm{a}$ & $25,0 \pm 0,3$ & $25,2 \pm 0,3$ & $24,8 \pm 0,3$ & $25,4 \pm 0,2 \mathrm{a}$ & $24,6 \pm 0,3 b$ \\
\hline $\begin{array}{l}\mathrm{LP}(\mathrm{cm}) \\
C W(\mathrm{~cm})\end{array}$ & $25,8 \pm 0,2$ & $25,0 \pm 0,3 b$ & $25,6 \pm 0,3 \mathrm{a}$ & $26,7 \pm 0,3 \mathrm{a}$ & $25,7 \pm 0,3$ & $25,8 \pm 0,3$ & $25,9 \pm 0,3$ & $26,6 \pm 0,2 \mathrm{a}$ & $25,0 \pm 0,3 b$ \\
\hline
\end{tabular}

Para cada fonte de variação, médias seguidas de mesma letra não diferem entre si $(P<0,05)$.

EC: escore corporal, CC: comprimento corporal, AA: altura anterior, AP: altura posterior, PT: perímetro torácico, PP: perímetro da perna, LG: largura da garupa, LP: largura do peito.

For each variation source, means followed by the same letter do not differ $(P<0.05)$.

BS: body score, BL: body length, FH: fore height, HH: hind height, TP: thoracic perimeter, LP: leg perimeter, RC: rump width, CW: chest width

com as fontes de variação analisadas. Os valores de comprimento corporal, altura anterior, altura posterior e perímetro

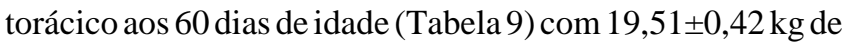
PV (Tabela 2) foram muito próximos dos obtidos por Yáñez et al. (2004) em cabritos Saanen com peso de $20 \mathrm{~kg}$ (55,8; 59,9; 56,9 e $61,5 \mathrm{~cm}$ respectivamente). Entretanto, neste estudo, a largura da garupa e a largura do peito foram superiores em $14,0 \mathrm{e} 16,6 \mathrm{~cm}$, respectivamente, às obtidas por esses autores. 
Os valores de comprimento corporal, altura anterior e perímetro toráxico obtidos aos 160 dias foram superiores aos obtidos por Mohammed \& Amin (1996) na raça Sahel com 16 semanas de idade $(67,4 ; 51,8$ e $49,1 \mathrm{~cm}$, respectivamente), possivelmente em virtude do pequeno porte dessa raça (38 e $33 \mathrm{~kg}$ para machos e fêmeas, respectivamente).

Todas as características biométricas, à exceção do escore corporal, aumentaram com idade de avaliação. A maior nota de escore corporal foi observada aos 60 dias (Tabela 9), entretanto, aos 90 dias, os animais apresentaram menor escore corporal, possivelmente em decorrência do estresse causado pelo desaleitamento, realizado aos 60 dias, que deve ter repercutido desfavoravelmente no peso aos 90 dias. Aos 120 dias, houve recuperação do escore corporal.

O escore corporal foi influenciado também pelo grupo racial, visto que a média dos $3 / 4$ Boer foi maior que a dos Alpinos, que não diferiu da média dos $1 / 2$ Boer. Esse resultado era esperado, pois a raça Boer tem sido selecionada para produção de carne. $\mathrm{O}$ escore corporal não diferiu entre machos e fêmeas.

O melhor escore corporal dos mestiços pode representar uma vantagem para o produtor na comercialização, principalmente quando comercializados vivos aos 60 dias de idade.

Os Alpinos foram superiores aos mestiços Boer quanto à altura anterior. A altura posterior, no entanto, foi maior nos Alpinos que nos 3/4 Boer, que não diferiram dos 1/2 Boer, indicando que os Alpinos são mais esguios, característica de animais com biótipo funcional leiteiro. Para todas as características biométricas, as estimativas obtidas para os machos foram maiores que as das fêmeas, exceto para escore corporal, o que comprova crescimento diferenciado nas fêmeas.

\section{Conclusões}

A participação da raça Boer em cruzamentos com a raça Alpina não promoveu melhora no desempenho dos cabritos, porém, se os animais forem comercializados vivos, o melhor escore corporal dos mestiços Boer pode proporcionar vantagens econômicas ao produtor.

Os machos foram superiores às fêmeas quanto ao peso ao nascimento, aos 60 e aos 90 dias de idade. Apresentaram ainda maior ganho de peso no intervalo de 30 a 60 dias e maiores medidas biométricas.

O menor custo de produção por quilo de cabrito (relativo à alimentação) foi observado aos 90 dias e pode ser atribuído à pior conversão alimentar observada a partir dessa idade. O custo da alimentação nesse sistema de criação foi elevado e pode acarretar dificuldades ao produtor na comercialização e obtenção de lucro.

\section{Literatura Citada}

DHANDA, J.S.; THAYLOR, D.G.; MC COSKER, J.E. et al. The influence of goat genotype on the production of Capretto and Chevon carcasses. 1 - Growth and carcass characteristics. Meat Science, v.52, p.355-361, 1999.

GONÇALVES, H.C.; SILVA, M.A.; REGAZZI, A.J. Fatores genéticos e de meio na idade ao primeiro parto de caprinos leiteiros. Revista Brasileira de Zootecnia, v.26, p.485-493, 1997.

GOONEWARDENE, L.A.; DAY, P.A.; PATRICK, N. et al. A Preliminary evaluation of growth and carcass traits in Alpine and Boer goat crosses. Journal Animal Science, v.78, p.229232, 1998

INSTITUTO BRASILEIRO DE GEOGRAFIA E ESTATÍSTICA IBGE. [2004]. Pesquisa Pecuária Municipal. Rio de Janeiro: IBGE. Disponível em: <www.ibge.gov.br/ibge/estatistica/ economia/agropecuaria/censoagro/default.shtm.> Acesso em: $05 / 03 / 2006$.

JOHNSON, D.D.; MC GONAN, C.H.; NURSE, G. et al. Breed type and sex effects on carcass traits, composition and tenderness of young goats. Small Ruminant Research, v.17, p.57-63, 1995.

KADIM, I.T.; MAHGOUB, O.; AL-AJMI, D.S. et al. An evaluation of the growth, carcass and meat quality characteristics of Omani goat breeds. Meat Science, v.66, p.203-210, 2003.

LUO, J.; SAHLU, T.; CAMERON, M. et al. Growth of Spanish, Boer $x$ Angora and Boer $\times$ Spanish goat kids fed milk replacer. Small Ruminant Research, v.36, p.189-194, 2000.

MAHGOUB, O.; LODGE, G.A. Growth and body composition in meat production of Omani Batina goats. Small Ruminant Research, v.19, p.233-246, 1996.

MOHAMMED, I.D.; AMIN, J.D. Estimating body weight from morphometric measurements of Sahel (borno white) goats. Small Ruminants Research, v.24, p.1-5, 1996

MOURAD, M.; FEHR, P.; HERVIER, J. Notaction de l'état corpore: a vos stylos. La chevre. Small Ruminant Research, v.175, p.39-42, 1989

NATIONAL RESEARCH COUNCIL - NRC. Nutrient requirements of goats. Washington, D.C.: 1981. 91p.

PRIETO, I.; GOETSCH, A.L.; BANSKALIEVA, V. et al. Effects of dietary protein concentration on postweaning growth of Boer crossbred and Spanish goat wethers. Journal of Animal Science, v.78, p.2275-2281, 2000.

REYNOLDS, L. Productivity of Boer x Small East African goats in Malawi. Journal Agricultural Research, v.23, p.1-8, 1985.

UNIVERSIDADE FEDERAL DE VIÇOSA - UFV. SAEG - Sistema de Análise Estatística e Genéticas. Viçosa, MG, 1997 (versão 7.0)

SINGH, R.N.; ACHARYA, R.M.; BISWAS, D.K. Evaluation of genetic and non-genetic factors affecting some economic traits in goats. Acta Agriculture Scand, v.20, p.10-14, 1970.

Van NIEKERK, W.A.; CASEY, N.H. The Boer goat 11. Growth, nutrient requirements, carcass and meat quality. Small Ruminant Research, v.1, p.355-368, 1988.

WALDRON, D.F.; HUSTON, J.E.; THOMPSON, P. et al. Growth rate, feed consumption and carcass measurements in Spanish and Boer x Spanish goats. Journal of Animal Science, v.73, p.253, 1995

YÁÑEZ, E.A.; RESENDE, K.T.; FERREIRA, A.C.D. et al. Utilização de medidas biométricas para predizer características de carcaça de cabritos Saanen. Revista Brasileira de Zootecnia, v.33, p.1564-1572, 2004 